\title{
PHENIX results on open heavy flavor production
}

\author{
Takashi Hachiya ${ }^{1,2, \star}$ for the PHENIX collaboration \\ ${ }^{1}$ Nara Women's University, Department of Physics, Nara, Japan \\ ${ }^{2}$ RIKEN BNL Research Center, Brookhaven National Laboratory, NY, USA
}

\begin{abstract}
PHENIX measures the open heavy flavor productions in $p+p, \mathrm{Cu}+\mathrm{Au}$, and $\mathrm{Au}+\mathrm{Au}$ collisions at $\sqrt{s_{N N}}=200$ and $510 \mathrm{GeV}$ using the silicon tracking detectors for mid- and forward rapidities. In Au+Au collisions, the nuclear modification of single electrons from bottom and charm hadron decays are measured for minimum bias and most central collisions. It is found that bottoms are less suppressed than charms in $p_{T}=3$ $5 \mathrm{GeV} / \mathrm{c}$ and charms in most central collisions are more suppressed than that in minimum bias collisions. In $p+p$ and $\mathrm{Cu}+\mathrm{Au}$ collisions, $J / \psi$ from $B$ meson decays are measured at forward and backward rapidities. The nuclear modification of $B$ mesons in $\mathrm{Cu}+\mathrm{Au}$ collisions is consistent with unity.
\end{abstract}

\section{Introduction}

Heavy quarks are useful probes to study the property of Quark -Gluon Plasma medium created in high energy heavy ion collisions. Heavy quark production is sensitive to the initial state effects because they are mainly produced via initial hard scattering. Once produced, they don't disappear by the strong interaction and they suffer final state effects such as energy loss and collective flow in QGP. Thus, their momentum and angular distributions are modified.

The PHENIX experiment previously measured a strong suppression of heavy quark production in $\mathrm{Au}+\mathrm{Au}$ collisions by the measurement of single leptons from the heavy quark decays[1,2]. The suppression of the charm quark was confirmed with the direct $D$ reconstruction by the STAR experiment[3]. To study the suppression of heavy quarks, especially bottoms, we installed the barrel and forward silicon vertex tracker (VTX and FVTX) in 2011 and 2012. The VTX and FVTX cover $|y|$ $<1.2$ and $1.2<y<2.2$ in rapidity and $2 \pi$ in azimuth. The VTX at mid-rapidity allows the statistical separation of electrons from bottom and charm decays using the track displacement from the collision vertex. In addition, $J / \psi$ from $B$ decays is a clean channel to measure the open bottom production. The FVTX at forward rapidity enables the extraction of $J / \psi$ from $B$ decays from prompt $J / \psi$.

In the proceedings, we report the recent results on bottom and charm electrons in central $\mathrm{Au}+\mathrm{Au}$ collisions and their nuclear modifications, and $J / \psi$ from $B$ decays in $p+p$ and $\mathrm{Cu}+\mathrm{Au}$ collisions at forward rapidity.

\footnotetext{
^e-mail: hachiya@cc.nara-wu.ac.jp
} 


\section{Single electron yields from bottom and charm decays in $\mathrm{Au}+\mathrm{Au}$ collisions}

We analyzed the dataset from $\mathrm{Au}+\mathrm{Au}$ collisions at $\sqrt{s_{N N}}=200 \mathrm{GeV}$ in 2014. The purpose of the analysis is the separation of electrons from bottom and charm hadron decays using the difference of their decay lengths. $B$ meson has longer decay length than that of $D$ mesons $\left(c \tau_{B^{0}} \approx 455 \mu m\right.$ and $c \tau_{D^{0}} \approx 123 \mu \mathrm{m}$ ). Electron identification was first performed using a ring-imaging Čerenkov detector and the energy-momentum matching. The distance of closest approach (DCA) of the electron to the collision vertex was then measured with the VTX. The DCA is proportional to the decay length, thus the shape of the DCA is broader for electrons from bottom decays than that from charm decays. Inclusive electrons contains not only the bottom and charm electrons but a large amount of background electrons. The main sources of these backgrounds are photon conversions, Dalitz decays of $\pi^{0}$ and $\eta$ as well as the detector effects such as mis-identified hadrons and high multiplicity background. Those backgrounds are estimated by the event driven method and the PHENIX detector simulations.

To separate the bottom and charm components, the unfolding method performs a simultaneous fitting to the DCA distributions and the invariant yield of electrons from inclusive heavy quark decays as described in the article[4]. Figure 1 shows the electron DCA distribution. The bottom and charm components obtained by the unfolding method are also plotted as well as the sum of the all background components.

The nuclear modification factor $\left(R_{A A}\right)$ for bottom electrons and charm electrons are calculated using the bottom electron fractions in $\mathrm{Au}+\mathrm{Au}$ and $p+p[5,6]$, and $R_{A A}$ of electrons from inclusive heavy quark decays[2]. Figure. 2 show $R_{A A}$ ' for minimum bias (MB) (left) and $0-10 \%$ central $\mathrm{Au}+\mathrm{Au}$ collisions (right). We found that bottom electrons are less suppressed than charm electrons decays for $p_{T}<4$ $\mathrm{GeV} / c$ and similarly suppressed for higher $p_{T}$ in both $0-10 \%$ central and MB collisions. The result is consistent with the expectation of the mass ordering of energy loss, $\Delta E_{c}>\Delta E_{b}$. In addition, charms in 0-10\% central collisions are more sup-

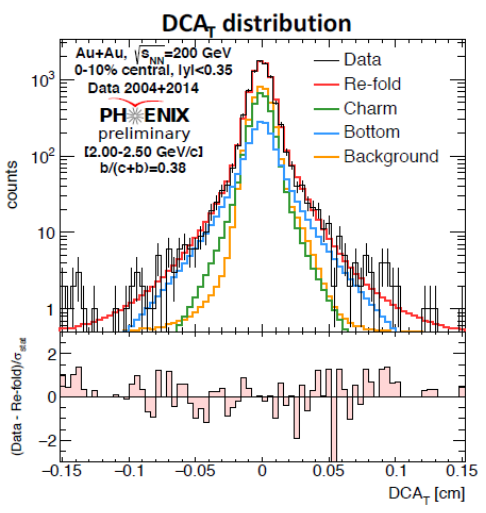

Figure 1. The electron DCA distribution for $0-10 \%$ central $\mathrm{Au}+\mathrm{Au}$ collisions. The bottom and charm components from the unfolding method are also plotted. pressed than that in MB collisions.

\section{$3 \mathrm{~J} / \psi$ from $B$ decays in $p+p$ and $\mathrm{Cu}+\mathrm{Au}$ collisions}

$J / \psi$ from $B$ meson decays is a clean measurement of open bottom production. The dataset used in the analysis were $p+p$ at $\sqrt{s}=200$ and $510 \mathrm{GeV}$ and $\mathrm{Cu}+\mathrm{Au}$ collisions in 2012 and 2015. B production in $p+p$ is good for testing perturbative QCD and provides an important baseline for the measurement in nuclear collisions. The advantage of asymmetric $\mathrm{Cu}+\mathrm{Au}$ collisions is to study QGP with different initial state effect by measuring at positive and negative rapidities.

PHENIX measures single muons from the decays of $B \rightarrow J / \psi(+X) \rightarrow \mu^{+}+\mu^{-}$at forward and backward rapidity. $J / \psi$ is reconstructed from unlike sign di-muon pairs within the invariant mass $2.8<M_{\mu \mu}\left(\mathrm{GeV} / c^{2}\right)<3.5$. FVTX measures DCA of single muons from $J / \psi$ decays. The DCA distribution of muons for $J / \psi$ from $B$ decay is broader than that of prompt $J / \psi$ and have longer tail in negative side because the detector acceptance at the negative side is wider, while the DCA for prompt $J / \psi$ is symmetric. 

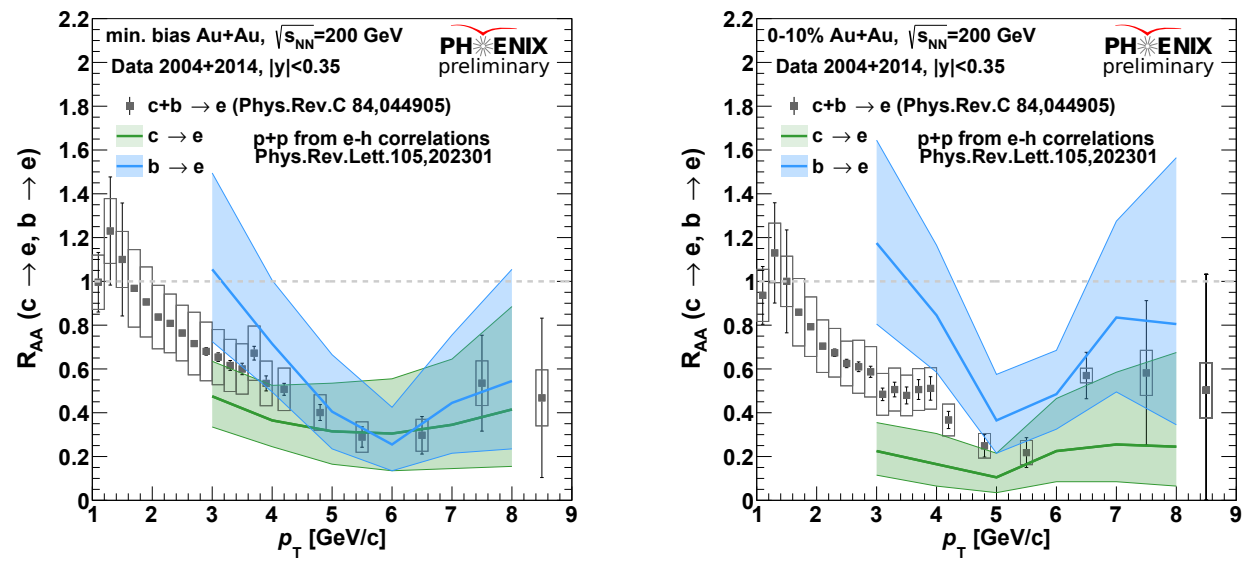

Figure 2. $R_{A A}$ for bottom and charm electrons for MB (left) and 0-10\% central Au+Au collisions (right)

Figure 3 shows the single muon DCA distribution from $J / \psi$ decays in $\mathrm{Cu}+\mathrm{Au}$ collisions. The DCA distribution is fit with the template DCA shapes of $J / \psi$ from $B$ decays and prompt $J / \psi$ to extract the $B$ decay components. The analysis procedure is described in detail[7]. The $J / \psi$ from $B$ decays and prompt $J / \psi$ from the fitting are also plotted.

Figure 4 shows the fraction of $J / \psi$ from $B$ decays relative to inclusive $J / \psi$ in $p+p$ and $\mathrm{Cu}+\mathrm{Au}$ collisions at $\sqrt{s}=200$ $\mathrm{GeV}$. By comparing with the FONLL calculation[8] for $\mathrm{J} / \psi$ from $B$ decays and Color Evaporation Model[9] for prompt $\mathrm{J} / \psi$, it is found that the calculation is consistent with the data in $p+p$. On the other hand, the fractions in $\mathrm{Cu}+\mathrm{Au}$ collisions are much larger than that in $p+p$.

The nuclear modification factor $R_{A A}$ of $J / \psi$ from $B$ decays and prompt $J / \psi$ is calculated using the $B$ rightarrow $/ \psi$ fractions in $\mathrm{Cu}+\mathrm{Au}$ and $p+p$, and the $R_{A A}$ of inclusive $J / \psi[10]$. Figure 5 shows these $R_{A A}$ as a function of rapidity. The $R_{A A}$ of $J / \psi$ of $B$ decays are consistent with unity within uncertainties for both $\mathrm{Cu}$ - and $\mathrm{Au}$ going directions even though is also consistent with a small enhancement from the initial state effect predicted by the EPS09 prediction[11].

In $510 \mathrm{GeV} p+p$ collisions, $d \sigma / d y(p p \rightarrow b \bar{b})$ is determined by the fraction of $J / \psi$ from $B$ decays and the inclusive $J / \psi$ cross section by the extrapolation of the PHENIX $200 \mathrm{GeV} p+p$ result[12]. The value of $d \sigma / d y(p p \rightarrow b \bar{b})$ is $3.57_{-2.22}^{+2.38} \mu \mathrm{b}$ at positive rapidity $\left(3.68_{-1.88}^{+2.08} \mu \mathrm{b}\right.$ at negative rapidity). These values are comparable with the FONLL calculation within large uncertainties.

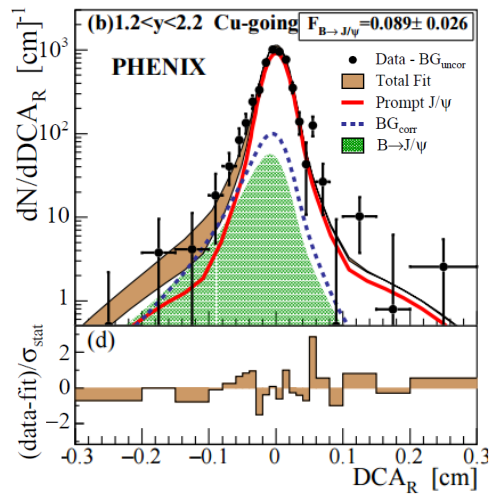

Figure 3. DCA distribution of muons from $J / \psi$ decays in $\mathrm{Cu}+\mathrm{Au}$ collisions. $B \rightarrow J / \psi$ component extracted by the fitting is plotted as well as the prompt $J / \psi$. 


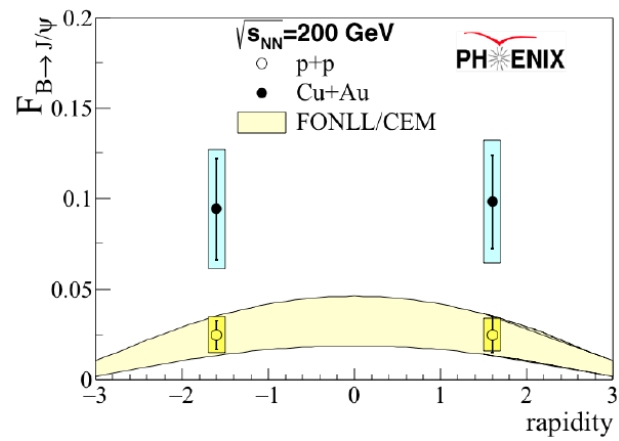

Figure 4. $B \rightarrow J / \psi$ fraction as a function of rapidity for $p+p$ and $\mathrm{Cu}+\mathrm{Au}$ collisions.

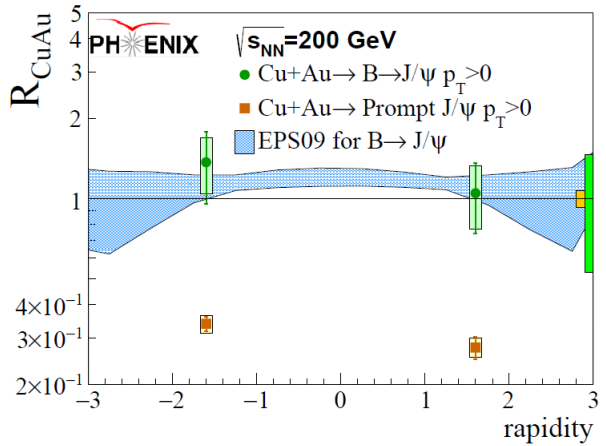

Figure 5. $R_{A A}$ of $J / \psi$ from $B$ decays and prompt $J / \psi$ as a function of rapidity.

\section{Summary}

PHENIX measured bottom and charm quark productions in $p+p, \mathrm{Cu}+\mathrm{Au}$, and $\mathrm{Au}+\mathrm{Au}$ collisions at $\sqrt{s_{N N}}=200$ and $510 \mathrm{GeV}$. The VTX and FVTX enables the statistical separation of the bottom and charm contributions. In $\mathrm{Au}+A u$, we studied the nuclear modification of bottoms and charms for $0-10 \%$ central collisions and MB collisions. The results show the less suppression of bottoms than that of charms. In addition, charms in $0-10 \%$ central collisions is more suppressed than that in $\mathrm{MB}$ collisions. From $J / \psi$ from $B$ decays by the FVTX at forward rapidity, the bottom yield in $p+p$ collisions is consistent with the the FONLL + CEM calculation. In $\mathrm{Cu}+\mathrm{Au}$ collisions, the nuclear modification of bottoms is consistent with the number of binary collision scaling for both positive and negative rapidities. These results provides important information about the QGP properties from new angle. The analyses with the significantly large amount of $\mathrm{Au}+\mathrm{Au}$ and $p+p$ data collected in 2014 to 2016 are underway. These new dataset should provide more precise measurement and quantitative constraints on the QGP properties.

\section{References}

[1] A. Adare et al. Phys. Rev. Lett. 98172301 (2007)

[2] A. Adare et al. Phys. Rev. C 84, 044905 (2011)

[3] L. Adamczyk et al. Phys. Rev. Lett. 113, 142301 (2014)

[4] A. Adare et al. Phys. Rev. C 93034904 (2016)

[5] A. Adare et al. Phys. Rev. Lett. 103082002 (2009)

[6] M. Aggarwal et al. Phys. Rev. Lett. 105202301 (2010)

[7] C. Aidala et al, arXiv:1702.01085

[8] M. Cacciari, M. Greco, and P. Nason, JHEP 05, 007 (1998)

[9] A. D. Frawley, T. Ullrich, and R. Vogt, Phys. Rept. 462,790 125 (2008)

[10] C. Aidala et al., Phys. Rev. C 90064908 (2014)

[11] K. J. Eskola, H. Paukkunen, and C. A. Salgado, JHEP 04, 065 (2009)

[12] C. Aidala et al., Phys. Rev. D 95092002 (2017) 